\section{New Trends in Myofunctional Therapy \\ Occlusion, Muscle and Posture}

Authors: Sabina Saccomanno,

Licia Coceani Paskay

Publisher: Edi.Ermes, Italy

Language: English

ISBN: 978-88-7051-629-6

Edition: 1/e

Publish Year: 2020

Pages: 330, illustrated

Price: $€ 68,00$

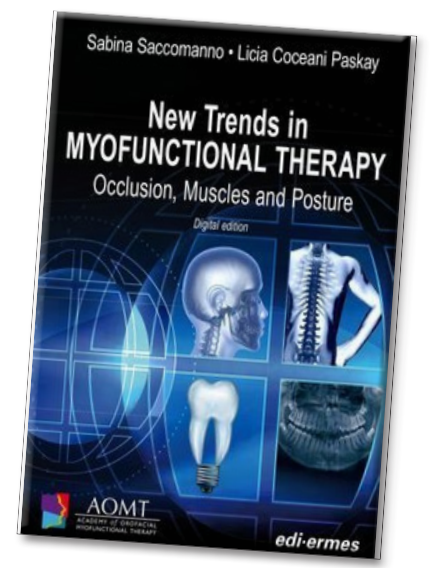

Marian-Vladimir

Constantinescu

$\mathrm{DDS}, \mathrm{MSc}, \mathrm{PhD}$

Holistic Dental \& Medical Institute

of Bucharest - ROPOSTURO

Bucharest, Romania

e-mail: dr.vladimir.constantinescu@gmail.com

In orthodontics and dento-facial orthopedics, a priority role is given to the oral function in both craniofacial growth and development, as well as in dental occlusion. Everyone is convinced that a young patient benefits from the orthodontist working in a medical team with specialists from different disciplines who follow the oral function evolution.

However, in current practice a young patient does not benefit from a holistic, inter and multidisciplinary evaluation, as a result of a concrete collaboration between doctors, dentists, orthodontists, speech therapists, myofunctional therapists, osteopaths and otorhinolaryngologists.

The book entitled "New Trends in Myofunctional Therapy" covers the experience of two editors, an orthodontist, Sabina Saccomanno, Professor Master in Clinical Orthodontics at the Catholic University of the Sacred Heart, Rome, Italy and a speech therapist Licia Coceani Paskay, Speech-Language Pathologist, with private practice, in Los Angeles, CA, USA, with 20 coauthors and 10 collaborators.

The volume has 15 chapters which approach the correlations between orofacial functions, occlusion and posture to better understand the appropriate diagnostic and therapeutic procedures that can be followed by the multidisciplinary medical team, addressing a young patient's global myofunctional disorders.

After an enumeration of medical records and multidisciplinary examination in holistic approach, an identification of malocclusion and a clinical and instrumental diagnosis of orofacial dysfunctions is made. Myofunctional therapy exercises and home-based myofunctional therapy and diary are presented in detail together with a myofunctional therapy protocol in alterations of the lingual frenulum. Orthodontic appliances in myofunctional therapy are eloquently illustrated by age groups. The book further focuses on unrelated topics in the field of orthodontics, such as orofacial pain, otitis media with effusion, obstructive sleep apnea syndrome, Down syndrome, and gloss-postural syndrome.

The book also provides practical tools, namely the Myofunctional Therapy Diary and Tongue Diary, as guides for a wide range of exercises, along with a video series explaining myofunctional therapy exercises.

The book confirms the validity of the Oro Myo Functional Therapy, proving to be a useful guide for understanding the importance of the functional balance between the craniofacial region and posture for doctors, dentists, orthodontists, speech therapists, myofunctional therapists, osteopaths and otolaryngologists. 